Archimer, archive institutionnelle de l'Ifremer Volume 52, Issue 3, April 2004, Pages 127-130 http://www.ifremer.fr/docelec/

\title{
Les polysaccharides microbiens d'origine marine et leur potentiel en thérapeutique humaine
}

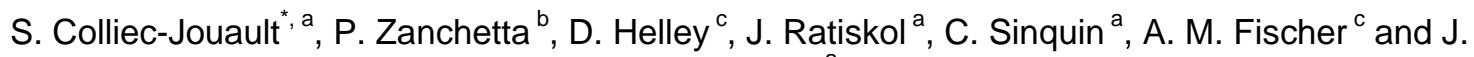 \\ Guezennec $^{a}$
}

\author{
${ }^{a}$ Laboratoire de biotechnologie et molécules marines (DRV/VP/BMM), Ifremer, rue de l'île-d'Yeu, BP \\ 21105, 44311, Nantes cedex 3, France \\ ${ }^{b}$ Service d'anatomo-pathologie, CHU Morvan, 29200, Brest, France \\ ${ }^{c}$ U428 Inserm, Faculté des sciences pharmaceutiques et biologiques, université Paris $\mathrm{V}, 4$, avenue de \\ l'Observatoire, 75270, Paris cedex 06, France
}

*: Corresponding author : sylvia.colliec.jouault@ifremer.fr

\begin{abstract}
Résumé : Des bactéries productrices d'exopolysaccharides (EPS) ont été isolées parmi la collection de l'IFREMER de micro-organismes provenant des écosystèmes hydrothermaux profonds. Plusieurs de ces EPS originaux ont d'ores et déjà été caractérisés. L'utilisation potentielle de ces EPS en tant que principes actifs pour la pharmacie a été explorée. Ainsi un EPS, secrété en conditions de laboratoire par une souche Vibrio diabolicus et présentant une analogie structurale avec l'acide hyaluronique, a été utilisé comme implant de comblement osseux. Cet EPS a montré un effet potentialisateur de la cicatrisation osseuse dans un modèle expérimental animal. Un second EPS biosynthétisé par la souche Alteromonas infernus et présentant une séquence osidique répétitive hautement ramifiée et riche en acide uronique a été modifié chimiquement pour obtenir un héparinomimétique. Cet EPS modifié, contrairement à son équivalent natif, présente alors des propriétés anticoagulantes. Ces EPS bactériens d'origine hydrothermale présentent une grande variabilité structurale qui peut être accentuée par modifications chimiques. Cette diversité structurale permet d'envisager l'utilisation de telles molécules à des fins thérapeutiques.
\end{abstract}

activité anticoagulante / biomimétique / cicatrisation osseuse / exopolysaccharide / sources hydrothermales

\footnotetext{
Summary : Exopolysaccharides produced by bacteria isolated from deep-sea hydrothermal vents : new agents with therapeutic potential. Bacterial polysaccharides offer fascinating potential applications for the pharmaceutical industry. Although many known marine bacteria produce exopolysaccharides (EPS), continuation in looking for new polysaccharide-producing micro-organisms is promising. Marine bacteria, isolated from deep-sea hydrothermal vents, have demonstrated their ability to produce in aerobic conditions, unusual EPS. With the aim of discovering biological activities, EPS presenting different structural features were studied. An EPS secreted by Vibrio diabolicus was evaluated on the restoration of bone integrity in experimental model and was demonstrated to be a strong bone-healing material. Another EPS produced by Alteromonas infernus was modified in order to obtain new heparin-like compounds. Unlike the native EPS, the resulting EPS presented anticoagulant properties as heparin. These EPS could provide biochemical entities with suitable functions for obtaining new drugs. They present original structural feature that can be modified to design compounds and improve their specificity.
}

anticoagulant activity /biomimetic / bone-healing / exopolysaccharides / hydrothermal vents 


\section{Introduction}

Grâce à ses moyens d'exploration des zones profondes, l'IFREMER a participé activement à l'étude des biotopes qui se sont développés au voisinage des sources marines hydrothermales chaudes $\left(>350^{\circ} \mathrm{C}\right)$ profondes où, en absence de lumière, les producteurs primaires de matière organique sont les bactéries. A l'issue de différentes campagnes océanographiques, l'IFREMER s'est constituée une collection de bactéries isolées de ces environnements qualifiés d'extrêmes. Cette collection compte à ce jour près de 1400 isolats. L'étude des applications biotechnologiques possibles de ces micro-organismes, qui se positionnent comme un champ d'investigation privilégié pour la recherche de biomolécules aux propriétés originales, a été développée. L'effort de recherche s'est porté tout d'abord sur des aspects d'écologie descriptive (caractérisation des souches, taxonomie, phylogénie...), l'obtention d'enzymes hautement spécifiques et plus récemment sur la recherche d'exopolysaccharides (EPS) bactériens d'intérêt industriel. Ces EPS pourraient alors soit se substituer aux polymères existants, soit trouver de nouvelles applications au regard de propriétés spécifiques liées à l’originalité de leur structure.

\section{Découverte de nouvelles bactéries productrices d'EPS}

Divers criblages effectués sur des échantillons récupérés lors des campagnes océanographiques ont mis en évidence la présence, dans ces milieux extrêmes, de bactéries aérobies mésophiles productrices, en conditions de laboratoire, d'exopolysaccharides (EPS) Plusieurs bactéries du genre Alteromonas ont été isolées [1, 2]. Parmi ces bactéries, une nouvelle espèce d'Alteromonas, provenant d'un fluide hydrothermal prélevé parmi une population de Riftia pachyptila dans la région du bassin de Guaymas, a été identifiée et l'appellation Alteromonas infernus lui a été donnée [3]. Cette souche a été déposée auprès de la Collection Nationale de Cultures de Micro-organisme (CNCM) à Paris. Une autre souche isolée d'un ver de Pompéi (Alvinella pompejana) a été caractérisée et l'appellation Vibrio diabolicus a été proposée. Cette souche a été également déposée auprès de la CNCM à Paris [4]. Chacune de ces souches est capable de synthétiser, en conditions de laboratoire un EPS dans le surnageant de culture sur milieu enrichi en glucose ou autre substrat carboné. Après moins de 72 heures de fermentation, les cellules bactériennes sont séparées du milieu de culture par centrifugation et le surnageant contenant les EPS excrétés est précipité à l'alcool. Les EPS précipités sont filtrés, lavés à l'alcool, et enfin séchés pour analyses et évaluations ultérieures de leurs principales caractéristiques physico-chimiques et activités biologiques.

\section{Caractérisation d'EPS originaux}

Un certain nombre d'EPS nouveaux ont d'ores et déjà été isolés et caractérisés. Plusieurs EPS produits par différentes bactéries du genre Alteromonas ont été décrits et été classés en 5 groupes différents $[1,2]$. Ces exopolysaccharides se caractérisent pour certains d'entre eux par des teneurs en acides uroniques comprises entre 10 et $40 \%$ en poids. Les teneurs en groupements sulfate sont comprises entre 10 et $20 \%$ en poids et ces exopolysaccharides à l'état natif sont tous de poids moléculaire élevé (>10 $\mathrm{g} / \mathrm{mol})$. L'EPS de haut poids moléculaire $\left(10^{6} \mathrm{~g} / \mathrm{mol}\right)$ produit par la souche Alteromonas infernus se caractérise par une forte teneur en oses neutres ( $57 \pm 4 \%$ en poids) et en oses acides ( $42 \pm 5 \%$ en poids) et présente un taux de sulfate de $10 \%$ environ. Les résultats des analyses par GC-MS, HPLC, RMN 2D (TOCSY, COSY, ROESY...) confirment la production par la bactérie Alteromonas infernus de deux EPS. L'EPS majoritaire est composé d'une séquence osidique répétitive de 9 résidus dont 5 résidus $\alpha$ et 4 résidus $\beta$. Un acide galacturonique est substitué en position 2 par un sulfate. L'unité répétitive compte 3 ramifications dont les résidus terminaux sont du glucose et du galactose. Ces ramifications sont portées par 2 acides glucuroniques et 1 acide galacturonique [5]. 
L'EPS produit par la souche Vibrio diabolicus présente des caractéristiques totalement différentes des autres EPS identifiés à ce jour puisque que possédant des teneurs élevées en osamines et présentant une forte analogie structurale avec l'acide hyaluronique [6]. Cet exopolysaccharide présente une teneur en osamines de $30 \pm 5 \%$ en poids, une teneur en oses acides de $32 \pm 5 \%$ en poids. Il est constitué d'acide glucuronique, de $\mathrm{N}$-acétyl-glucosamine et de $\mathrm{N}$-acétyl-galactosamine. Il ne présente pas d'unité osidique sulfatée et son poids moléculaire est de l'ordre de $810^{5} \mathrm{~g} / \mathrm{mol}$. Sa structure a été récemment décrite, la séquence osidique répétitive est linéaire et constituée de 4 résidus [7]:

$\rightarrow 3$ )- $\beta$-D-GlcpNAc-( $1 \rightarrow 4)$ - $\beta$-D-GlcpA-( $1 \rightarrow 4)$ - $\beta$-D-GlcpA-(1 $\rightarrow 4)-\alpha-D-G a l p N A c-(1 \rightarrow$

\section{Utilisation d'un EPS excrété par l'espèce Vibrio diabolicus en cicatrisation osseuse.}

L'EPS de haut poids moléculaire produit par fermentation de la souche Vibrio diabolicus a été utilisé comme implant de comblement osseux dans un modèle expérimental [8]. Des lésions osseuses au niveau des deux lobes pariétaux ont été pratiquées dans la calotte crânienne chez le rat. Chez un même animal, le défaut de taille critique réalisé dans le pariétal droit est comblé par de l'EPS sous sa forme native ou du collagène (matériau pris comme référence) tandis que le défaut de taille critique réalisé dans le pariétal gauche est laissé vacant. Après 15 jours tous les animaux traités avec cet exopolysaccharide montrent une cicatrisation complète à $96 \%$ au niveau du défaut comblé et partielle à $30 \%$ au niveau du défaut non traité. Parallèlement les animaux traités au collagène montrent une très faible cicatrisation au niveau des deux lésions : 18 \% pour la lésion comblée par du collagène et 14 $\%$ pour la lésion vacante. Du point de vue histologique, aucune réaction inflammatoire n’est observée, l'EPS utilisé n'est plus décelable et l'os néoformé en 15 jours est parfaitement structuré. Les fibres de collagène sont orientées, les ostéoblastes recouvrent les surfaces osseuses, des ostéocytes sont présents. Cet os est histologiquement normal. Une néovascularisation intense est également observée et le tissu conjonctif cutané n’a pas proliféré de façon anarchique. En outre les cicatrices cutanées sont d'excellente qualité et sans phénomène prolifératif.

De nombreux travaux expérimentaux montrent l'intérêt des facteurs de croissance pour accélérer la repousse osseuse dans des défauts de taille critique au niveau du crâne chez le rat [9], de la mandibule chez le chien [10] ou encore des os longs chez le mouton [11]. La capacité des protéoglycanes et de certains polysaccharides à contrôler les cytokines comme les facteurs de croissance et l'interféron-gamma par crinopexie (potentialisation de l'activité, régulation de la biodisponibilité et de la stabilité) est bien connue [12]. L’EPS considéré dans cette étude doit probablement interagir avec des médiateurs solubles mais également avec des composants matriciels ou cellulaires et ainsi favoriser le phénomène de cicatrisation.

\section{Préparation d'un héparinomimétique par modification d'un EPS excrété par l'espèce Alteromonas infernus.}

En vue de développer de nouvelles substances bioactives, des produits de faible masse molaire ont été préparés par hydrolyse chimique ou dépolymérisation radicalaire. Les EPS natifs $\left(10^{6} \mathrm{~g} / \mathrm{mol}\right)$ et partiellement dépolymérisés $(<30000 \mathrm{~g} / \mathrm{mol})$ étant inactifs dans les tests de coagulation, la sulfatation des produits a été réalisée en vue d’obtenir des héparinomimétiques ou " heparin-like” [13]. Une fraction de bas poids moléculaire (24 000 $\mathrm{g} / \mathrm{mol})$ et homogène en masse avec une polydispersité $(\mathrm{Mw} / \mathrm{Mn})$ de 1,3 a été obtenue à partir de l'EPS natif par dépolymérisation radicalaire. Le dérivé sulfaté ( $40 \%$ sulfate) obtenu est anticoagulant. Dans les tests de coagulation, ce dérivé est 2,5 moins actif que l’héparine de bas poids moléculaire et 7,5 fois moins actif que l'héparine non fractionnée, ce qui diminuerait le risque hémorragique potentiel en cas d'utilisation thérapeutique. L'étude de son mécanisme d’action a montré que, tout comme l’héparine, ce dérivé diminue la quantité de 
thrombine générée après activation de la coagulation par la phase contact (voie endogène). En revanche, il retarde de manière beaucoup plus importante que l'héparine cette génération de thrombine. De plus, par électrophorèse d'affinité, il a été montré que toutes les chaînes polysaccharidiques se lient fortement à l'antithrombine (inhibiteur physiologique de la thrombine et des sérine-protéases de la coagulation en général), contrairement à l'héparine non fractionnée dont seulement $1 / 3$ des chaînes (contenant le pentasaccharide) se lient à l'antithrombine. L'électrophorèse montre également qu'une faible proportion des chaînes présentent une forte affinité pour le deuxième cofacteur de l'héparine, autre inhibiteur physiologique spécifique de la thrombine [14].

\section{Conclusion}

La diversité structurale des polysaccharides quelle que soit leur origine (animale, végétale ou microbienne) confère à ces macromolécules de nombreuses activités biologiques. Ainsi grâce à leurs propriétés interactives et régulatrices, les polysaccharides participent au contrôle de l'activité cellulaire (prolifération, différenciation, adhésion et migration) mais également de l'activité de nombreuses enzymes. De telles molécules aux multiples fonctions spécifiques peuvent être exploitées en thérapeutique. La production, en condition de laboratoire, de polysaccharides bactériens (exopolysaccharides ou EPS) d'intérêt industriel constitue un axe nouveau des biotechnologies marines. Les EPS bactériens présentent des atouts comme l'absence de dépendance vis à vis d'aléas climatiques, écologiques et politiques pouvant affecter la qualité, le coût et l'approvisionnement de leurs homologues extraits d'organes d'animaux, d'algues ou de plantes. De plus les possibilités d'agir sur les conditions de fermentation (sources de carbone, température, aération, $\mathrm{pH} . .$. ), en vue d'optimiser la production mais aussi de modifier le polymère produit, jouent en faveur de la fermentation bactérienne. Agir sur les conditions de fermentation ou encore sur le matériel génétique des micro-organismes constituent donc des axes de recherche auxquels vient naturellement s'ajouter celui de la découverte de nouveaux micro-organismes capables de synthétiser ces polysaccharides.

Les exemples d'activité biologique mis en évidence au cours de ces études confirment le potentiel thérapeutique de ces polysaccharides microbiens d'origine marine. Ces EPS sécrétés par les bactéries marines d'origine hydrothermale présentent une grande variabilité structurale qui peut être encore accentuée par modifications chimiques. Cette diversité structurale permet d'envisager la préparation de molécules présentant des fonctions multiples mais spécifiques pouvant être exploitées à des fins thérapeutiques. 


\section{Références}

1. Guezennec J, Pignet P, Raguenes G, Deslandes E, Lijour Y, Gentric E. Preliminary chemical characterization of unusual eubacterial exopolysaccharides of deep-sea origin. Carbohydr Polym 1994 ; 24:287-94.

2. Vincent P, Pignet P, Talmont F, Bozzi L, Fournet B, Guezennec J. Production and characterization of an exopolysaccharide excreted by a deep-sea hydrothermal vent bacterium isolated from the polychaete annelid Alvinella pompejana. Appl Environ Microb 1994 ; 60:4134-41.

3. Raguenes GH, Peres A, Ruimy R, Pignet P, Christen R, Loaec M, Rougeaux H, Barbier G, Guezennec JG. Alteromonas infernus sp. nov., a new polysaccharide-producing bacterium isolated from a deep-sea hydrothermal vent. J Appl Microbiol 1997 ; 82:422-30.

4. Raguenes G, Christen R, Guezennec J, Pignet P, Barbier G. Vibrio diabolicus sp. nov., a new polysaccharide-secreting organism isolated from a deep-sea hydrothermal vent polychaete annelid, Alvinella pompejana . Int J Syst Bacteriol 1997 ; 47:989-95.

5. Roger O. Etude d'oligosaccharides bioactifs issus d'exopolysaccharides bactériens : obtention, caractérisation et relation structure/fonction. [Thèse]. Université Paris 13 ; 2002.

6. $\quad$ Deming JW. Deep ocean environmental biotechnology. Curr Opin Biotechnol 1998 ; 9:283-7.

7. Rougeaux H, Kervarec N, Pichon R, Guezennec J. Structure of the exopolysaccharide of Vibrio diabolicus isolated from a deep-sea hydrothermal vent. Carbohydr Res 1999 ; 322:40-5.

8. Zanchetta P, Lagarde N, Guezennec J. A new bone-healing material: a hyaluronic Acid-like bacterial exopolysaccharide. Calcif Tissue Int 2003 ; 72:74-9.

9. Arnaud E, De Pollak C, Meunier A, Sedel L, Damien C, Petite H. Osteogenesis with coral is increased by BMP and BMC in a rat cranioplasty. Biomaterials 1999 ; 20:1909-18.

10. Toriumi DM, Kotler HS, Luxenberg DP, Holtrop ME, Wang EA. Mandibular reconstruction with a recombinant bone-inducing factor. Functional, histologic, and biomechanical evaluation. Arch Otolaryngol Head Neck Surg 1991 ; 117:1101-12.

11. Gerhart TN, Kirker-Head CA, Kriz MJ, Holtrop ME, Hennig GE, Hipp J, Schelling SH, Wang E. Healing segmental femoral defects in sheep using recombinant human bone morphogenetic protein. Clin Orthop 1993 ; 317-26.

12. Nathan C, Sporn M. Cytokines in context. J Cell Biol 1991 ; 113:981-6.

13. Guezennec J, Pignet P, Lijour Y, Gentric E, Ratiskol J, Colliec-Jouault S. Sulfation and depolymerization of a bacterial exopolysaccharide of hydrothermal origin. Carbohydr Polym $1998 ; 37: 19-24$.

14. Colliec Jouault S, Chevolot L, Helley D, Ratiskol J, Bros A, Sinquin C, Roger O, Fischer AM. Characterization, chemical modifications and in vitro anticoagulant properties of an exopolysaccharide produced by Alteromonas infernus. Biochim Biophys Acta 2001 ; 1528:141-51. 